\title{
An Unusual Presentation of Eosinophilic Gastroenteritis: Case Report and Literature Review
}

\author{
Mohammed A. Alzahrani*,, ${ }^{*}$, Ali M. Assiri ${ }^{2}$, Saif A. Alqahtani ${ }^{3}$, Sabah N. Nemri ${ }^{4}$, and Abdullah M. \\ Alshehri $^{4}$ \\ ${ }^{1}$ Internal Medicine Department, King Khalid University - KSA \\ ${ }^{2}$ Internal Medicine Department, Najran University - KSA \\ ${ }^{3}$ Internal Medicine Department, King Khalid University, Abha, KSA \\ ${ }^{4}$ Assir Central Hospital, Abha, KSA
}

\begin{abstract}
Eosinophilic gastroenteritis [EGE] is a rare disease of the gastrointestinal tract characterized by eosinophilic infiltration of the bowel wall and variable gastrointestinal manifestation. The signs and symptoms of EGE are related to the extent of bowel involved with eosinophilic infiltration whether mucosa; muscle; and / or subserosa. Peripheral eosinophilia may or may not be present. Diagnosis requires a high index of suspicion and exclusion of other causes of peripheral eosinophilia. We report a case of a young woman who presented with recurrent abdominal pain and ascites. Diagnosis of EGE was made based on clinical, laboratory, radiological and histological criteria. The patient demonstrated overlap between different subtypes of EGE and had an excellent response to steroid treatment.
\end{abstract}

Keywords: Eosinophilic gastroenteritis [EGE], ascites, abdominal pain.

\section{INTRODUCTION}

Eosinophilic gastroentritis [EGE] is a rare disease and a member of a family of eosinophilic gastroenteritis disorders [EGIDs] [1]. EGE was first described by Kaijser et al in 1937 [1, 2]. EGE is characterized by an eosinophil driven inflammation of digestive tract, and the clinical presentation ranged from non -specific gastrointestinal complaints such as abdominal pain, nausea, vomiting and diarrhea to specific findings such as malabsoption , protein losing enteropathy, intestinal obstruction, eosinophilic ascites and pleural effusion [3]. The clinical features of EGE relates to the extent of bowel involved with eosinophilic inflammation ranging from mucosa, muscle, and /or subserosa [4]. Definitive diagnosis requires histological demonstration of eosinophilic infiltration into the gastrointestinal wall or high eosinophil count in ascetic fluid, absence of extra gastrointestinal eosinophilic infiltration, and exclusion of other diseases that can present in a similar fashion [5].

\section{CASE}

A 30-year-old Saudi female, presented at our hospital, with a one week history of abdominal distension. There was no history of hematemesis, malaena or change in bowel habits. There was no history of weight loss, fever, recurrent mouth ulcers, malar rash or joint pain, nor was there was any history of allergy to drugs or food. One day before admission, she went to a polyclinic where an abdominal ultrasound

*Address correspondence to this author at the Department of Medicine, College of Medicine, King Khalid University, P. O. Box 641, Abha, 61421, Kingdom of Saudi Arabia; Tel: 00966-504586134; Fax: 00966- 72247570; E-mail: dr_mohdaz@hotmail.com was performed and which revealed mild to moderate ascites. She had visited another hospital 3 years previously with a complaint of recurrent epigastric pain and she underwent upper gastrointestinal endoscopy with no apparent unusual diagnosis. Helicobacter pylori were confirmed by serology and she was subjected to triple medications [omeprazole, clarihromycin, amoxicillin]. Also, she has microcytic hypochromic anemia for 4 years and she was on iron supplementation. At Emergency Room, the patient was generally well and the vital signs were normal .No lymphadenopathy was detectable. Chest, cardiovascular and central nervous system examinations were normal. Abdominal examination showed positive shifting dullness and no organomegaly. Her initial investigations revealed leukocyte count of 16.9 $\mathrm{x} 10^{3} / \mathrm{L}$, hemoglobin count of $15 \mathrm{~g} / \mathrm{dl}$ and platelet count of $200 \times 10^{3} / \mathrm{L}$. Coagulation profile, urea and electrolytes were within normal range. Peripheral blood smear revealed a high eosinophilic count [40\%]. The anti amoebic titer was negative and stool microscopy was negative for cyst and ova. Anti- nuclear antibody, rheumatoid factor and HIV serology were negative. Diagnostic paracentesis of the ascetic fluid showed low SAAG [serum / ascites albumin gradient] and absolute eosnophilia but no malignant cells .Computed tomography of the abdomen with contrast showed mild ascites and thickening of the duodenum (Fig. 1). Upper gastrointestinal endoscopy showed mild duodenitis. Biopsies of the duodenum revealed marked eosinophilic infiltration (Fig. 2).

After excluding the possibilities of a parasitic disease, autoimmune and malignancy, EGE was made based on clinical, laboratory, radiological and histological criteria. A daily dose of steroid [prednisolone $30 \mathrm{mg}$ ] was given. One week later, the patient recovered from her symptoms. The white 


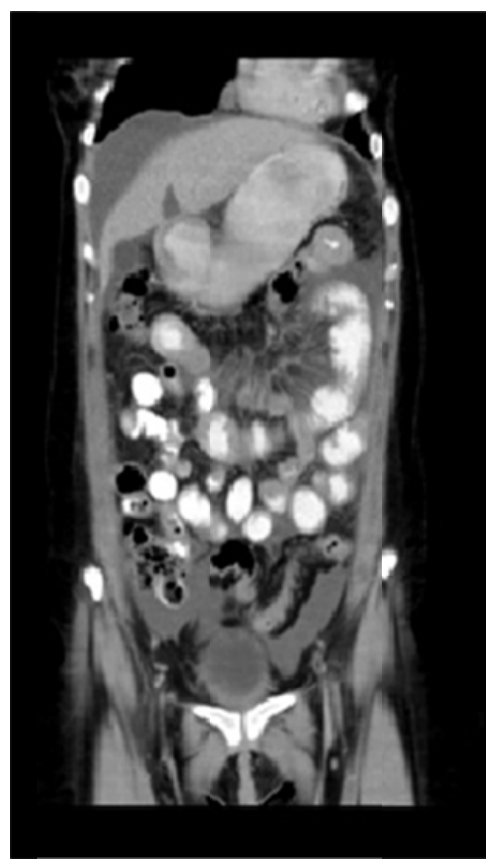

Fig. (1). Computed tomography of the abdomen showed non obstructive bowel pattern with increase in small bowel thickening and moderate ascetic fluid.

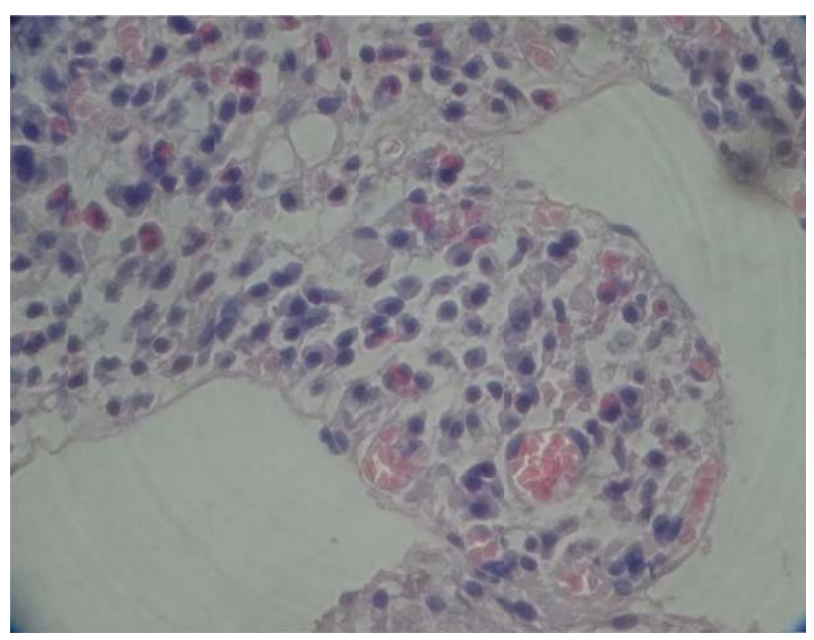

Fig. (2). Duodenal biopsy showed marked eosinophilic infiltration.

blood cell count was $9.5 \times 10^{3} / \mathrm{L}$ with $2 \%$ eosinophils. Steroid treatment was tapered and terminated after 6 weeks. Two months later, the patient had recovered from EGE and follow up by ultrasound of abdomen showed presence of no ascites.

\section{DISCUSSION}

Primary EGE is defined as a disorder that affects the gastrointestinal tract with eosinophilic - rich inflammation in the absence of other known causes of eosinophilia, such as drug reaction, parasitic infection, or malignancy [1]. EGE can affect patients of any age, but typically it is found in the third through fifth decade of life with a male predominance $[6,7]$. The pathological process may occur in any part of the gastrointestinal tract with; stomach and duodenum most commonly involved. However, the patients may have various clinical presentations depending on the region of the gastro-intestinal tract involved and the depth of the bowel wall involvement. EGE is therefore classified into mucosal, muscular and subserosal subtypes with, considerable overlap $[4,6]$.

Mucosal EGE, which is the most common EGE subtype, presents with abdominal pain, nausea, vomiting, diarrhea, anaemia and weight loss secondary to malabsorption or protein losing enteropathy $[4,5,8,9]$. Muscularis EGE, often manifests as gastric outlet or small bowel obstruction [4-6, 8]. Subserosal EGE, presents with isolated ascites or ascites in combination with symptoms characteristic of mucosal or muscularis EGE $[5,6,8,10]$. The pathogenesis and etiology of the disease is not well understood. Diagnostic evaluation of EGE is based on a high index of clinical suspicion with peripheral eosinophilia, the absence of an identified cause of eosinophilia, and the exclusion of eosinophilic involvement in organs other than gastro-intestinal tract.

Talley et al. [6] identified three main diagnostic criteria of EGE: [1] the presence of gastrointestinal symptoms, [2] biopsies demonstrating eosinophilic infiltration of one or more areas of the gastrointestinal tract, and [3] absence of parasitic or extra-intestinal disease. However, definitive diagnosis requires histological evidence of eosinophilic infiltration of $>20$ eosinopils per high power field on microscopic examination. In subserosal EGE, abdominal paracentesis demonstrates a sterile fluid with high eosinophilic count $[5,6,11,12]$. Our patient suffered from recurrent abdominal pain for about three years resulting in three hospital visits and upper gastrointestinal endoscopic intervention. She was also found to have ascites, and a high eosinophilic count was found in peripheral blood smear and ascetic fluid and other causes of eosinophilia were excluded. Biopsies were taken from stomach and duodenum which showed eosinophilic infiltration of mucosa and submucosal layers of the intestinal wall. Thus this patient fulfilled the criteria for the diagnosis of EGE, and has overlap between EGE subtypes.

Radiological imaging tests are not helpful in diagnosis of EGE and do not have a pathognomonic appearance. The most common computed tomography finding is thickening of the intestinal wall and occasionally, localized lymphadenopathy. Ascites is usually detected in patients with subserosal layer involvement [5, 11-13]. Endoscopic evaluation may demonstrate changes in the gastrointestinal mucosa varying from normal appearance to severe inflammation, with erosion, exudates, furrowing polyps, mucosal rings and stricture formation [5, 6, 8, 14].

The ideal treatment of EGE is to identify and remove the causative agents if they exist but in adults, the causative agents are not usually evident $[15,16]$. Treatment with steroids is the mainstay of managing EGE. Clinical improvement usually occurs within two weeks regardless the layer of bowel involved and usage of prednisolone should be tapered over two to four weeks. However, some patients require prolonged therapy [up to several months] to produce resolution of symptoms [8, 11]. Long- term prognosis of patients with EGE is generally good $[8,11]$. Adult patients may experience relapse of the disease and may need chronic low dose of steroids or transition to budesonide or leukotriene antagonist [17-19]. 


\section{ACKNOWLEDGEMENTS}

None declared.

\section{CONFLICT OF INTEREST}

The authors confirm that this article content has no conflicts of interest.

\section{REFERENCES}

[1] Rothenberg ME. Eosinophilic gastrointestinal disorders [EGID]. J Allergy Clin Immunol 2004; 113: 11.

[2] Kaijser R. Zurkenntnis der allergischen affeclctionen des verdauungskkanals von stondpunkt des chirugen aus. Langenbecks Arch Klin Chir Ver Dtsch Z Chir 1937; 188: 36-64.

[3] Guajardo JR, Plotnick LM, Fende JM, Collins MH, Putanam PE, Rothenberg ME. Eosinophil- associated gastrointestinal disorders: a worldwide web based registry. J Pediatr 2002; 141(4): 571-81.

[4] Klein NC, Hargrove RL, Sleisenger MH, Jeffries GH. Eosinophilic gastroenteritis. Medicine [Baltimore] 1970; 49: 299-319.

[5] Baig MA, AbdullQadir, Rashid J. A review of eosinophilic gastroenteritis. J Natl Med Assoc 2006; 98: 1616-9.

[6] Talley NJ, Shorter RG, Phillips SF, Zinsmeister AR. Eosinophilic gastroenteritis: a clinicopathological study of patients with disease of the mucosa, muscle layer, and subserosal tissues. Gut 1990; 31: 54-8.

[7] Foroughi S, Foster B, Kim N, et al. Anti-IgE treatment of eosinophil-associated gastrointestinal disorders. J Allergy Clin Immunol 2007; 120: 594-601.
[8] Cello JP. Eosinophilic gastroenteritis--a complex disease entity. Am J Med 1979; 67: 1097-104.

[9] Friesen CA, Kearns GI, Andre L, et al. clinical efficacy and pharmacokinetics of montelukast in dyspeptic children with duodenal eosinophilia. J Pediatr Gastroenterol Nutr 2004; 38: 343-51.

[10] Chen MJ, Chu CH, Lin SC, et al. Eosinophilic gastroenteritis: clinical experience with 15 patients. World J Gastroenterol 2003; 9: 2813-6.

[11] Lee CM, Changchien CS, Chen PC, et al. Eosinophilic gastroenteritis: 10 years experience. Am J Gastroenterol 1993; 88: 70-4.

[12] Lee M, Hodges WG, Huggins TL, Lee EL. Eosinophilic gastroenteritis. South Med J 1996; 89: 189-94.

[13] Blackshaw AJ, Levison DA. Eosinophilic infiltrates of the gastrointestinal tract. J Clin Pathol 1986; 39: 1-7.

[14] Fox VL. Eosinophilic esophagitis: endoscopic findings. Gastrointest Endosc Clin N Am 2008; 18: 45-57.

[15] Gonsalves N, Doerfler B, Yang G, et al. A prospective clinical trial of six food elimination diet or elemental diet in the treatment of adults with eosinophilic gastroenteritis [abstract]. Gastroenterology 2009; 136: S1861.

[16] Kagalwalla AF, Sentongo TA, Ritz S, et al. Effect of six-food elimination diet on clinical and histologic outcomes in eosinophilic esophagitis. Clin Gastroenterol Hepatol 2006; 4: 1097-102.

[17] Tan AC, Kruimel JW, Naber TH. Eosinophilic gastroenteritis treated with non-enteric-coated budesonide tablets. Eur J Gastroenterol Hepatol 2001; 13: 425-7.

[18] Elsing C, Placke J, Gross-Weege W. Budesonide for the treatment of obstructive eosinophilic jejunitis. Z Gastroenterol 2007; 45: 1879.

[19] Neustrom MR, Friesen C. Treatment of eosinophilic gastroenteritis with montelukast. J Allergy Clin Immunol 1999; 104: 506.

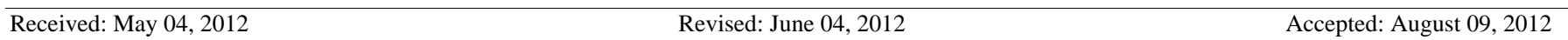

(C) Alzahrani et al.; Licensee Bentham Open.

This is an open access article licensed under the terms of the Creative Commons Attribution Non-Commercial License (http://creativecommons.org/licenses/by-nc/3.0/) which permits unrestricted, non-commercial use, distribution and reproduction in any medium, provided the work is properly cited. 